\title{
Osteopetrosis and thalamic hypomyelinosis with synaptic degeneration in DAP12-deficient mice
}

\author{
Tomonori Kaifu, ${ }^{1}$ Jin Nakahara, ${ }^{2}$ Masanori Inui, ${ }^{1}$ Kenichi Mishima, ${ }^{3}$ \\ Toshihiko Momiyama, ${ }^{4}$ Mitsuji Kaji, ${ }^{5}$ Akiko Sugahara, ${ }^{1}$ Hisami Koito, ${ }^{1}$ \\ Azusa Ujike-Asai, ${ }^{1}$ Akira Nakamura, ${ }^{1}$ Kiyoshi Kanazawa, ${ }^{6}$ Kyoko Tan-Takeuchi, ${ }^{2}$ \\ Katsunori Iwasaki, ${ }^{3}$ Wayne M. Yokoyama, ${ }^{7}$ Akira Kudo, ${ }^{6}$ Michihiro Fujiwara, ${ }^{3}$ \\ Hiroaki Asou, ${ }^{2}$ and Toshiyuki Takai ${ }^{1}$
}

\begin{abstract}
${ }^{1}$ Department of Experimental Immunology and Core Research for Evolutional Science and Technology (CREST) Program of the Japan Science and Technology Corporation (JST), Tohoku University, Sendai, Japan

${ }^{2}$ Department of Neurobiology, Tokyo Metropolitan Institute of Gerontology, Tokyo, Japan

${ }^{3}$ Department of Neuropharmacology, Faculty of Pharmaceutical Sciences, Fukuoka University, Fukuoka, Japan

${ }^{4}$ Division of Cerebral Structure, National Institute for Physiological Sciences, Okazaki, Japan

${ }^{5}$ Department of Clinical Oncology, Institute of Development, Aging and Cancer, Tohoku University, Sendai, Japan

${ }^{6}$ Department of Life Science, Tokyo Institute of Technology, Yokohama, Japan

${ }^{7}$ Division of Rheumatology, Department of Medicine, Howard Hughes Medical Institute, Washington University

School of Medicine, St. Louis, Missouri, USA
\end{abstract}

Deletions in the DAP12 gene in humans result in Nasu-Hakola disease, characterized by a combination of bone fractures and psychotic symptoms similar to schizophrenia, rapidly progressing to presenile dementia. However, it is not known why these disorders develop upon deficiency in DAP12, an immunoreceptor signal activator protein initially identified in the immune system. Here we show that DAP12-deficient (DAP12-/-) mice develop an increased bone mass (osteopetrosis) and a reduction of myelin (hypomyelinosis) accentuated in the thalamus. In vitro osteoclast induction from $D A P 12^{-/-}$bone marrow cells yielded immature cells with attenuated bone resorption activity. Moreover, immature oligodendrocytes were arrested in the vicinity of the thalamus, suggesting that the primary defects in $D A P 12^{-/-}$ mice are the developmental arrest of osteoclasts and oligodendrocytes. In addition, the mutant mice also showed synaptic degeneration, impaired prepulse inhibition, which is commonly observed in several neuropsychiatric diseases in humans including schizophrenia, and aberrant electrophysiological profiles in the thalami. These results provide a molecular basis for a unique combination of skeletal and psychotic characteristics of Nasu-Hakola disease as well as for schizophrenia and presenile dementia.

J. Clin. Invest. 111:323-332 (2003). doi:10.1172/JCI200316923.

\section{Introduction}

Nasu-Hakola disease (1-5) involves a unique combination of skeletal symptoms and CNS disorders. About 160 patients have been diagnosed with Nasu-Hakola,

Received for publication September 17, 2002, and accepted in revised form October 31, 2002.

Address correspondence to: Toshiyuki Takai, Department of Experimental Immunology, Institute of Development, Aging and Cancer, Tohoku University, Seiryo 4-1, Sendai 980-8575, Japan. Phone: 81-22-717-8501; Fax: 81-22-717-8505;

E-mail: tostakai@idac.tohoku.ac.jp.

Tomonori Kaifu, Jin Nakahara, and Masanori Inui contributed equally to this work.

Conflict of interest: The authors have declared that no conflict of interest exists.

Nonstandard abbreviations used: triggering receptor expressed on myeloid cells (TREM); immunoreceptor tyrosine-based activation motif (ITAM); $\gamma$-aminobutyric acid (GABA); miniature inhibitory postsynaptic current (mIPSC); lymphokine-activated killer cell (LAK cell); microcomputed tomography (micro-CT); tartrate-resistant acid phosphatase (TRAP); receptor activator of the NF- $\mathrm{KB}$ ligand (RANKL); myelin basic protein (MBP); postnatal day $(\mathrm{P})$. mostly in Japan and Finland (5). Nasu-Hakola patients develop systemic bone cysts and psychotic symptoms similar to schizophrenia such as loss of social inhibitions, personality changes, euphoria, and hallucination, rapidly progressing to presenile dementia at age 30-40. Nasu-Hakola is a fatal disorder with no specific treatment, and death before age 50 usually results from chest infection. The mutation found in Finnish patients is a $5.3-\mathrm{kb}$ deletion in the DAP12 locus (also known as KARAP or TYROBP), while the defect found in Japanese patients is a single-nucleotide deletion in the third exon of the gene (6). Both mutations result in nonfunctional DAP12, a membrane adapter protein found initially in cells of the immune system such as NK cells and myeloid cells $(7,8)$.

In the immune system, DAP12 is expressed as a disulfide-linked homodimer that associates with various immunoreceptors, many of whose ligands are unknown, including TREM2 (triggering receptor expressed on myeloid cells-2) $(9,10)$. DAP12 harbors an immunoreceptor tyrosine-based activation motif (ITAM) also 
found in other immunoreceptor adapters such as the $\mathrm{Fc}$ receptor common $\gamma$ chain. Tyrosine phosphorylation of ITAM of these immunoreceptor adapters by Src-family kinase is the primary step for delivery of activation signaling to the cell interior. In addition to the cells in the immune system, Paloneva et al. (6) observed expression of mRNA for DAP12 in human brain samples and in rodent CNS cells. Recent gene targeting studies of DAP12 in mice $(11,12)$ demonstrated a nonredundant role of the molecule in the immune system, but did not describe any pathology in bones or brain of the young $D A P 12^{-/-}$mice. There has also been speculation that the expression of DAP12 in the monocyte-macrophage lineage might provide a link between lesions in the brain and bone (6), because both microglial cells in the CNS and osteoclasts in bone tissue share a common differentiation pathway with macrophages. In this context, it was recently demonstrated that DAP12 plays a critical role in myeloid cell differentiation (13). The inability of microglial cells to remove apoptotic tissue in the brain may give rise to the CNS pathogenesis. On the other hand, bone cysts might result from chronic dysfunction of osteoclasts, resulting in a defective bone remodeling process (6). Recent identification of the second NasuHakola gene, TREM2, encoding an immunoglobulin-like receptor that associates with DAP12, has provided a clue to why DAP12 signaling is necessary for the maintenance of normal bone and CNS functions (14).

Here we show that as early as 6 weeks of age, $D A P 12^{-/-}$ mice exhibited osteopetrosis in which bone resorption was impaired due to attenuated activity of osteoclasts. Mature DAP12 $2^{-/-}$mice also exhibited thalamus-accentuated hypomyelinosis with synaptic degeneration, possibly due to arrested oligodendrocyte development. Electrophysiological examinations revealed the aberrant profiles of $\gamma$-aminobutyric acid (GABA) receptormediated miniature inhibitory postsynaptic currents (mIPSC's) in the thalamus of DAP12-/- mice. Consistent with this observation, the mutant mice exhibited a reduced startle response as well as an impaired prepulse inhibition to acoustic stimuli, indicating the deficit in sensorimotor gating that is commonly observed in several neuropsychiatric diseases such as schizophrenia in humans.

\section{Methods}

Gene targeting of the DAP12 locus. DAP12-/- mice were generated in the $129 / \mathrm{SvJ}$ and C57BL/6 hybrid background as described (15). All experiments were performed on 3 - to 48-week-old mice with appropriate littermate controls housed and bred in the Animal Unit of The Institute of Development, Aging and Cancer (IDAC; Tohoku University, Sendai, Japan), an environmentally controlled and specific pathogen-free facility, according to guidelines for experimental animals defined by the facility. Animal protocols were reviewed and approved by the IDAC Animal Studies Committee.

Immunoblot analysis. Lymphokine-activated killer (LAK) cells and bone marrow-derived cultured mast cells were prepared according to standard protocols. Cell lysates were separated by SDS-PAGE using 15\% gels and transferred onto PVDF membranes (Millipore Corp., Bedford, Massachusetts, USA). The membranes were incubated with rabbit anti-DAP12 antiserum followed by probing with peroxidase-conjugated goat anti-rabbit IgG (Kirkegaard and Perry Laboratories, Gaithersburg, Maryland, USA).

Bone histology and osteoclast culture. Microcomputed tomography (micro-CT) was performed using MCTCB100MF (Hitachi Medical Corp., Tokyo, Japan). Skeletal tissues were fixed in paraformaldehyde, decalcified in EDTA, dehydrated in ethanol, and embedded in paraffin. Sections were stained with hematoxylin and eosin or tartrate-resistant acid phosphatase (TRAP) using a TRAP staining kit (TaKaRa Biomedicals, Ohtsu, Japan). For electron microscopy, femur samples were postfixed in $1 \% \mathrm{OsO}_{4}$ and $0.8 \% \mathrm{~K}_{4} \mathrm{Fe}(\mathrm{CN})_{6}$ in $0.1 \mathrm{M}$ sodium cacodylate buffer and embedded in Epon 812 (TAAB Laboratories Equipment Ltd., Berkshire, England). Ultrathin sections $(85 \mathrm{~nm})$ were examined with an H-7100 system (Hitachi Hi-Technologies Co., Tokyo, Japan). For culturing osteoclasts, bone marrow cells were cultured with M-CSF $(50 \mathrm{ng} / \mathrm{ml})$ in $\alpha$-MEM containing $10 \%$ FCS at $5 \times 10^{5}$ cells $/ \mathrm{ml}$. After 3 days, cells were harvested and further cultured with mouse M-CSF and soluble receptor activator of NF- $\mathrm{KB}$ ligand (RANKL) or TNF- $\alpha\left(50 \mathrm{ng} / \mathrm{ml}\right.$ each) at $5 \times 10^{4}$ cells $/ \mathrm{ml}$. After 5 days in culture, cells were fixed and stained for TRAP. To determine the bone resorption activity of osteoclasts, bone marrow cells were cultured as above on a dentine slice for 6 days. The slice was stained with hematoxylin. The pit areas on the slice were measured under a light microscope. To visualize actin ring formation, bone marrow cells were cultured as above on glass coverslips for 6 days, fixed with $4 \%$ paraformaldehyde, and permeabilized with $0.1 \%$ Triton X-100. Cells were incubated with Texas red-conjugated phalloidin and viewed with a confocal microscope (Nippon Bio-Rad Laboratories, Tokyo, Japan).

Immunohistochemistry and electron microscopy of brain. Brains were fixed overnight with $95 \%$ ethanol and 5\% acetic acid, and then paraffinated, sliced $10 \mu \mathrm{m}$ thick, and deparaffinated. We used the following primary antibodies: rabbit anti-mouse myelin basic protein (MBP) (Nichirei Corp., Tokyo, Japan), anti-glial fibrillary acidic protein (anti-GFAP) (DAKO Japan Co., Kyoto, Japan), anti-neurofilament (anti-NF) (a gift from M. Ogawa, RIKEN Brain Science Institute, Wako, Japan), and anti-DAP12. Immunostaining was performed as described (16) using a DAB staining kit (DAKO Japan Co.). Counterstain was methyl green (Wako Pure Chemical Industries Ltd., Osaka, Japan). Nissl (cresyl violet) staining was performed according to standard procedures. For electron microscopy, cerebrum samples were postfixed in $1 \% \mathrm{OsO}_{4}$ in 0.1 $M$ sodium cacodylate buffer and embedded in Epon 812. Ultrathin $(85 \mathrm{~nm})$ cross sections of cerebra were examined as above. 
Primary neural cell cultures. Primary cultures of mouse brain neural cells were obtained as described $(17,18)$. $\mathrm{BALB} / \mathrm{c}$ mouse brains were dissociated in an isotonic buffer (19) containing $0.25 \%$ trypsin and $0.001 \%$ DNase I. Cells were washed, sieved through $70-\mu \mathrm{m}$ nylon mesh, and seeded in T75 flasks $\left(2.5 \times 10^{7}\right.$ cells/flask). After 10 days, the flasks were shaken on a rotary shaker for 30 minutes to obtain the loosely attached microglia. Then oligodendrocytes and their progenitors were removed from the flasks by shaking for 24 hours. Astrocytes remaining in the culture flasks were treated with trypsin and collected. Oligodendrocytes and their progenitors $\left(3 \times 10^{5}\right.$ cells $\left./ \mathrm{ml}\right)$ were reacted at $4^{\circ} \mathrm{C}$ for 20 minutes with $\mathrm{O} 1, \mathrm{O} 4$, or $\mathrm{A} 2 \mathrm{~B} 5$ antibodies attached to magnetic beads (BioMag; PerSeptive Biosystems Inc., Framingham, Massachusetts, USA) and then collected by a magnetic separator. To ensure that $\mathrm{O}-2 \mathrm{~A}$ progenitor cells were obtained, antibodyconjugated beads were used in reverse order of differentiation (first O1, then O4, and finally A2B5). For neurons, dissociated cells of the embryonic cerebrum were seeded on dishes coated with poly-L-lysine $\left(2 \times 10^{5}\right.$ cells $\left./ \mathrm{cm}^{2}\right)$ and cultured in DMEM-Ham's F12 (1:1) containing 5\% FCS. After 1 day, the medium was replaced with serum-free DMEM-F12 supplemented with insulin $(5 \mu \mathrm{g} / \mathrm{ml})$, sodium selenite (30 nM), transferrin $(50 \mu \mathrm{g} / \mathrm{ml})$, hydrocortisone $(10 \mathrm{nM})$, triiodothyronine $(30 \mathrm{nM})$, and cytosine arabinoside $(10 \mu \mathrm{M})(20)$. Cells were harvested after 6 days.

RT-PCR analysis. Total RNA was extracted from $\mathrm{BALB} / \mathrm{c}$ mouse brain with Trizol reagent (Life Technologies Inc., Rockville, Maryland, USA). First-strand cDNA was synthesized with random primers (TaKaRa Biomedicals). Thirty cycles of PCR were carried out with Ex Taq DNA polymerase (TaKaRa Biomedicals), with denaturation at $93^{\circ} \mathrm{C}$ for 40 seconds, annealing at $64^{\circ} \mathrm{C}$ for 40 seconds, and elongation at $72^{\circ} \mathrm{C}$ for 40 seconds. The following synthetic oligonucleotides were used for amplification of DAP12 cDNAs: 5'-CTGGTGCCTTCTGTTCCTTC-3' and 5'-CCTCTGTGTGTTGAGGTCAC-3'.

Acoustic startle response and prepulse inbibition. Responses of mice were measured in the SR-Lab system (San Diego Instruments Inc., San Diego, California). Mice were placed into cylinders 10 minutes prior to the initial startle stimuli and only background noise (65 dB) was offered during this acclimation period. The amplitude of the startle response was recorded for $100 \mathrm{~ms}$ from the onset of the startle stimulus. The maximum amplitude of the startle response was used as the variable. For measuring the acoustic startle response, eight different types of startle stimuli $(70,75,80,85,90,100$, 110 , and $120 \mathrm{~dB}, 20$-ms broadband burst) were used. Each stimulus was repeated 5 times in the same order. The trials were separated by an interval of an average of 40 seconds. For the acoustic prepulse inhibition, each session consisted of seven different types of stimuli: no stimulus, startle stimulus trials only, and a prepulse preceding the startle stimulus. The "no stimulus" trials were performed to measure baseline movement in the cylinders. The two "startle stimulus only" trials consisted of 20 -ms startle stimuli of either 100 or 120 dB. Finally, there were four combinations of acoustic prepulse plus acoustic startle stimuli. Each 20 -ms prepulse stimulus (either 70 or $80 \mathrm{~dB}$ ) was presented 100 ms before acoustic startle stimulus (either 100 or 120 $\mathrm{dB})$. The seven different types of stimuli were presented nine times in random order. The trials were separated by an interval of an average of 40 seconds. Percentage prepulse inhibition of a startle response was calculated as: (1 - startle response on prepulse preceding to the startle pulse stimulus/startle response on startle pulse stimulus alone) $\times 100$. Data were analyzed using two-way ANOVA with repeated measures followed by the Student $t$ test for differences between groups when the overall significance was determined in repeated measures of two-way ANOVA.

Electrophysiology. After decapitation of mice under halothane anesthesia, coronal slices (300 $\mu \mathrm{m}$ thick) containing laterodorsal thalamic nucleus were cut using a tissue slicer (DTK-1000; Dosaka EM Co., Kyoto, Japan) in ice-cold oxygenated standard artificial cerebrospinal fluid with low $\mathrm{Ca}^{2+}(0.5 \mathrm{mM})$ and high $\mathrm{Mg}^{2+}$ $(6 \mathrm{mM})(21)$. Slices were incubated at room temperature $\left(21-25^{\circ} \mathrm{C}\right)$ in standard artificial cerebrospinal fluid for 1 hour before recording. Whole-cell recordings were made using a patch-clamp amplifier (Axopatch 200B; Axon Instruments Inc., Foster City, California, USA). Patch pipettes were pulled from standard-walled borosilicate glass capillaries $(1.5 \mathrm{~mm}$ outer diameter; Clark Electromedical Instruments, Reading, United Kingdom) and had a resistance of 3-6 $\mathrm{M} \Omega$ when filled with the internal solution, which consisted of $140 \mathrm{mM}$ CsCl, $9 \mathrm{mM} \mathrm{NaCl}, 1 \mathrm{mM}$ Cs-EGTA, $10 \mathrm{mM}$ Cs-HEPES, and $2 \mathrm{mM} \mathrm{Mg-ATP}$, with $\mathrm{pH}$ adjusted with $1 \mathrm{M} \mathrm{CsOH}$. The access resistance was monitored by measuring the capacitative transients generated in response to a hyperpolarizing voltage step $(5 \mathrm{mV}, 25 \mathrm{~ms})$ from a holding potential of $-60 \mathrm{mV}$. No correction was made for the liquid junction potential (calculated to be 5.0 $\mathrm{mV}$ by pClamp7 software; Axon Instruments Inc.). Experiments were carried out at room temperature $\left(21-25^{\circ} \mathrm{C}\right)$. Data were stored using a DAT recorder (PC204AX; Sony Corp., Tokyo, Japan). mIPSC's were filtered at $2 \mathrm{kHz}$, digitized at $20 \mathrm{kHz}$ using pClamp8 software (Axon Instruments Inc.), and analyzed with $\mathrm{N}$ software (provided by Stephen F. Traynelis, Emory University, Atlanta, Georgia, USA) (21).

\section{Results}

Targeted mutation of DAP12 gene in mice. DAP $12^{-/-}$mice were generated by standard gene-targeting methods that resulted in the deletion of a putative promoter and exons 1-3 of DAP12 (Figure 1a). Mice with the -/genotype had no DAP12 protein detected by immunoblot analysis of LAK cells (Figure 1, b and c). Expression of the DAP10 gene, which lies only $0.1 \mathrm{~kb}$ from DAP12 and also encodes a DAP12-related cell-surface adapter protein $(22,23)$, was not impaired by our 


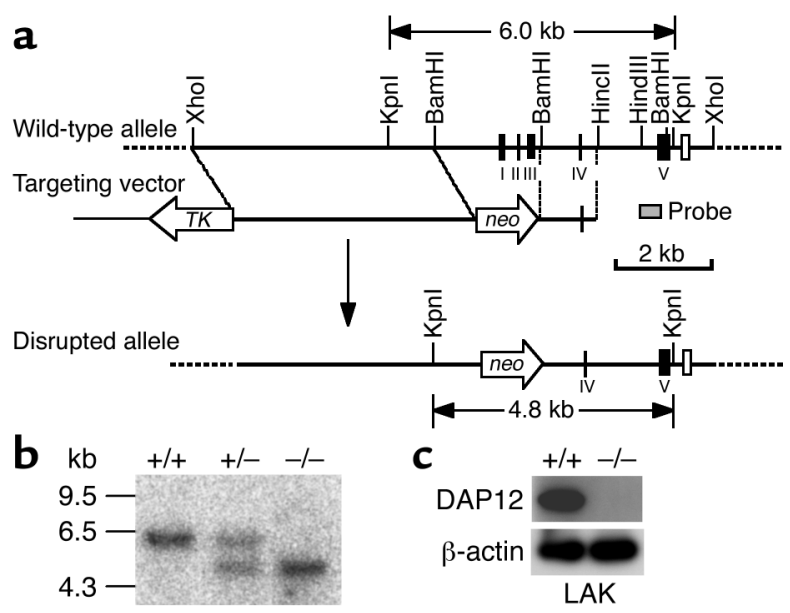

\section{Figure 1}

Targeted mutation of the DAP12 gene in mice. (a) Schematic illustration of the DAP12 locus (top), targeting vector (middle), and disrupted allele (bottom). Exons 1-5 (I-V) are shown (filled boxes). Exon 4 of the DAP10 gene is also shown (open box). The neor (neo) and thymidine kinase (TK) genes and the probe (shaded box) for Southern blotting are shown. A $5.1-\mathrm{kb} \mathrm{BamHI}$ fragment containing the promoter region and exons 1-3 of the DAP12 gene was replaced by a neor cassette; $5.1 \mathrm{~kb}$ and $1.2 \mathrm{~kb}$ of homologous sequences flanking the neor cassette were retained. (b) Southern blot analysis to confirm the genotype of DAP12 wild-type $(+/+)$, heterozygote (+/-), and knockout (-/-) offspring. Genomic DNA isolated from the tail tips of offspring of heterozygous intercrosses were digested with $K p n l$ and probed. The wild-type allele migrates as a $6.0-\mathrm{kb}$ fragment; the disrupted allele, $4.8 \mathrm{~kb}$. (c) Immunoblot analysis. Protein extracts from IL-2-induced LAK cells $\left(5 \times 10^{5}\right.$ cell equivalent per lane) of wild-type or DAP12-/- mice were probed with rabbit anti-DAP12 antiserum (1:1,000 dilution) or $\beta$-actin antibody. DAP1 2 protein was not detected in the cells from $D A P 12^{-/-}$mice. gene-targeting strategy as assessed by immunoblot analysis (data not shown).

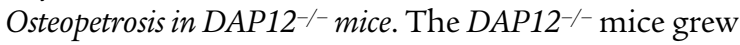
normally and were fertile and without any gross behavioral abnormalities up to at least 24 months of age. To analyze possible abnormalities in skeletal tissues, we compared radiograms, micro-CT scan images, and the histology of skeletons from wild-type and DAP12-/ mice of various ages. While radiograms of the whole bodies of 12-week-old mice did not show obvious differences apart from a slight increase in bone density of the mutant mice, especially in tail bones and the meta- physeal regions of the femurs (not shown), micro-CT scan 3D imaging revealed an increase in trabecular bone mass of the tibia from 40-week-old DAP12--- mice (Figure 2a). Hematoxylin and eosin staining of longitudinal sections of the distal metaphyseal regions of the femurs showed that $D A P 12^{-/-}$mice 6 weeks of age and older possessed significantly higher amounts of bone trabecula than littermate controls, but 3-week-old DAP12--mice did not (Figure 2, b and c), indicating that osteopetrosis becomes evident as DAP12-/- mice grow.

As is the case with other osteopetrotic mice such as Csf1 $1^{o p / o p}$ mice $(24,25)$, osteopetrosis in DAP12 $2^{-/-}$mice

\section{Figure 2}

Osteopetrosis in DAP12-/- mice. (a) Micro-CT 3D images of tibial metaphyses of 40-week-old wild-type and DAP12-/- mice. DAP12 ${ }^{-/-}$mice showed increased bone mass. (b) Hematoxylin and eosin staining of metaphysis sections from 6-week-old wild-type and mutant mice. (c) Percentages of trabecular bone volume per total tissue volume (BV/TV) of the femurs from wild-type (black bars) and mutant mice (white bars) at various ages. ${ }^{*} P<0.05 . n=3$. (d) The numbers of $\mathrm{TRAP}^{+}$multinucleated osteoclasts (OCs) in 6-week-old wild-type and DAP12-/- mice are not significantly different. (e) Transmission electron micrographs of in vivo osteoclasts from wild-type and $D A P 12^{-/-}$mice do not show significant differences. $D A P 12^{-/-}$osteoclasts displayed a ruffled border $(\mathrm{rb})$, a complex membrane structure, which is usually associated with active bone matrix resorption, and a clear zone $(\mathrm{cz})$ attached to the bone matrix. $\mathbf{a}$
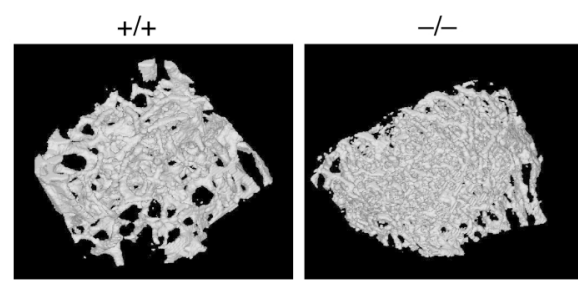

Micro-CT 3D images

b
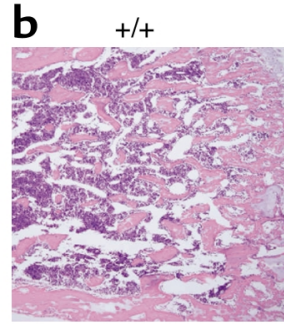

$-1-$
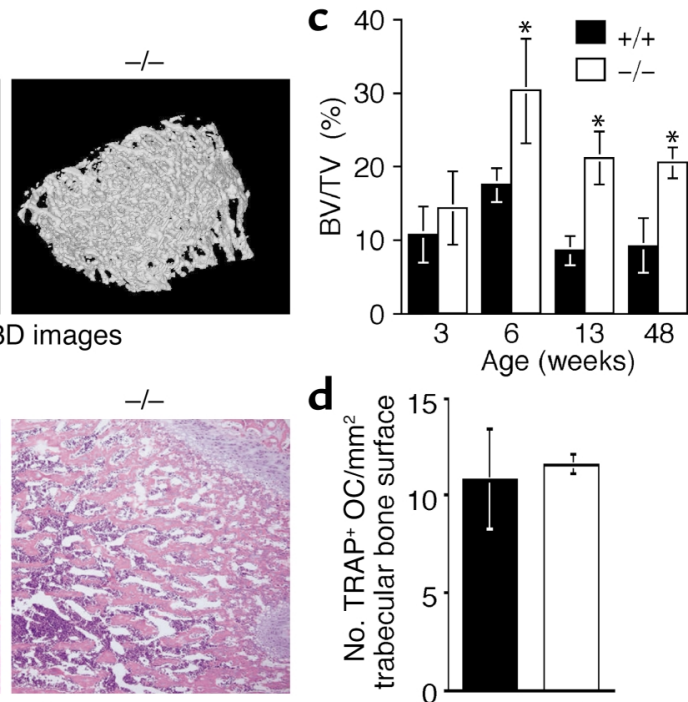

e

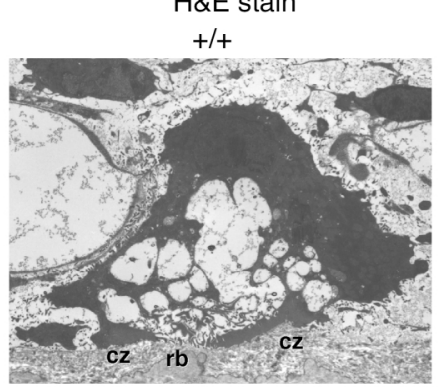

d

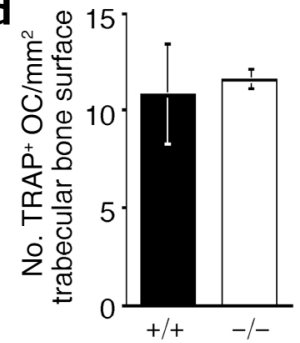
$-1-$

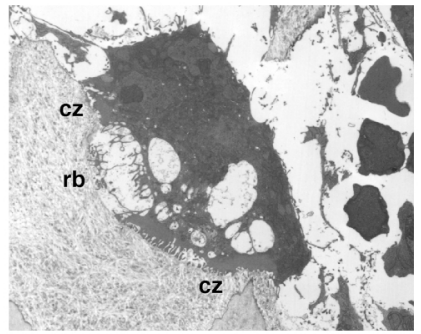


may be due to failed osteoclast development and impaired bone remodeling at these sites. Comparable numbers of multinucleated osteoclasts (seen with TRAP staining) per unit trabecular area between femurs of 6-week-old DAP12-/- and control mice (Figure $2 \mathrm{~d}$ ) suggested impairment in osteoclast function rather than a reduction of multinucleated osteoclasts in $D A P 12^{-/-}$mice. However, ultrastructural analysis of $D A P 12^{-/-}$bone tissue revealed a comparable morphology of osteoclasts from $D A P 12^{+/+}$and $D A P 12^{-/-}$mice, although $D A P 12^{-/-}$osteoclasts in vivo appeared to be somewhat smaller (Figure 2e).

$D A P 12$ is required for functional osteoclasts in vitro. Multinucleated, mature osteoclasts with bone-resorption activity can be induced in vitro from bone marrow cells in the presence of M-CSF (26) and RANKL (27). Additionally, TNF- $\alpha$ was recently shown to be just as potent as RANKL in that it can promote osteoclastogenesis in the presence of M-CSF (28). Taking advantage of this culture system, we attempted to assess developmental or functional differences in osteoclasts induced in vitro from bone marrow cells of DAP12-/- and wild-type mice. Even in the presence of sufficient M-CSF and RANKL or TNF- $\alpha$, the induction of multinucleated, $\mathrm{TRAP}^{+}$osteoclasts was significantly hampered in $D A P 12^{-/-}$bone marrow cells, while wild-type cells yielded a number of multinucleated osteoclasts (Figure 3, a and b). After induction with RANKL or TNF- $\alpha$, $D A P 12^{-/-}$osteoclasts with more than two nucleoli were reduced to, respectively, roughly one-third or one-half the number found in the wild-type control (Figure 3b). Importantly, the morphology of the multinucleated osteoclasts induced in vitro from $D A P 12^{-/-}$bone marrow cells was quite different from that of osteoclasts from wild-type mice in that the DAP12-/- cells were devoid of an actin ring, which is characteristic of mature and functional osteoclasts and is necessary for tight adhesion of osteoclasts to bone matrix (Figure 3c). These mutant osteoclasts had a significantly reduced ability to form resorptive pits on the dentin surface, while wild-type osteoclasts made many pits (Figure 3d). Immunoblot analysis verified the expression of DAP12 protein in wild-type osteoclasts induced in vitro (Figure 3e). Thus, in the in vitro culture system, DAP12 is required for normal development of multinucleated, mature osteoclasts with bone resorptive activity. Although neither the number nor morphology of osteoclasts in vivo was significantly different between wild-type and $D A P 12^{-/-}$mice (Figure 2, $\mathrm{d}$ and e), it is conceivable that the bone resorptive activity of osteoclasts is reduced in the mutant mice.

Hypomyelinosis in DAP12-/- mice. Maturational and functional defects in DAP12-/- osteoclasts suggest the possibility that microglial cells may not be developed in the brain because of the common differentiation pathway of microglial cells, macrophages, and osteoclasts. Immunohistochemical analyses, including staining for microglia (anti-F4/80 antibody), neuronal cells (Nissl staining), neurofilaments (anti-NF antibody), and astroglia (anti-GFAP antibody) of cerebral

\section{Figure 3}

In vitro development of osteoclasts from DAP12-/- mice is impaired. (a) TRAP staining of cultured osteoclasts induced in vitro in the presence of M-CSF and RANKL. Reduction of multinucleated TRAP ${ }^{+}$osteoclasts is evident in cells from DAP12-/mice. Original magnification: $\times 100$. Multinucleated osteoclasts are developed from DAP12-/- bone marrow cells as seen at higher magnification (original magnification: $\times 400$ ). (b) Comparison of the number of multinucleated (having more than two nuclei) TRAP ${ }^{+}$osteoclasts induced by RANKL (left) or TNF- $\alpha$ (right). ${ }^{*} P<0.05,{ }^{*} P<0.01 . n=3$. (c) Actin ring formation by osteoclasts. DAP12-/- osteoclasts did not form actin rings. (d) Formation of resorption pits by osteoclasts induced in wild-type or DAP12-/- mice. DAP12-/- osteoclasts did not form significant pits. ${ }^{*} P<0.05 . n=3$. (e) Immunoblot analysis of DAP12 expression in osteoclasts induced from bone marrow cells. Osteoclasts ( $10 \mu \mathrm{g}$ protein per lane) from wildtype mice showed DAP12 expression, although at lower levels than were measured in bone marrow-derived cultured mast cells (BMMCs) $(10 \mu \mathrm{g}$ protein per lane) from wild-type mice.
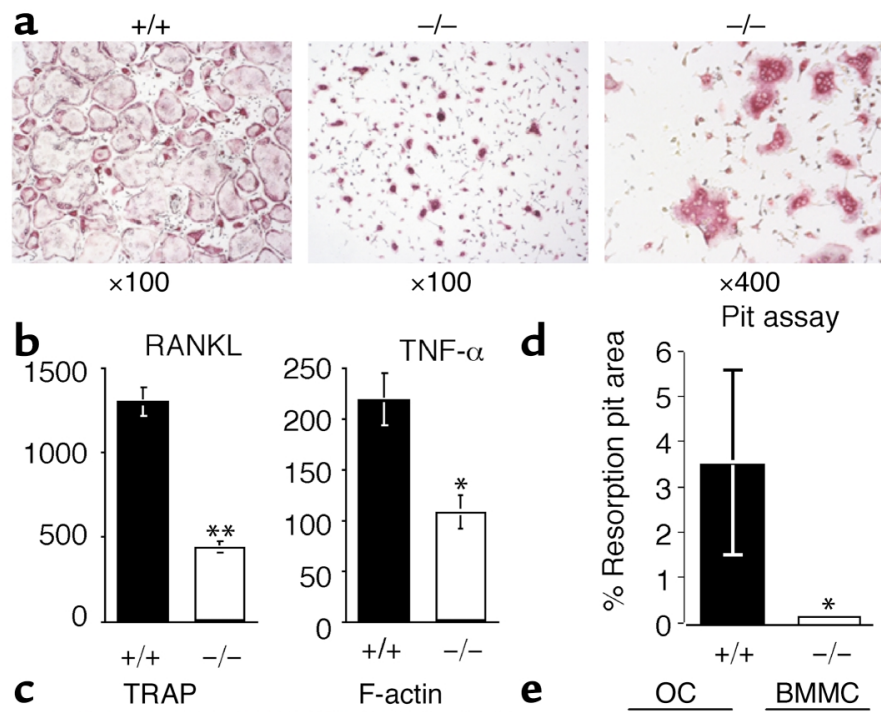

Pit assay

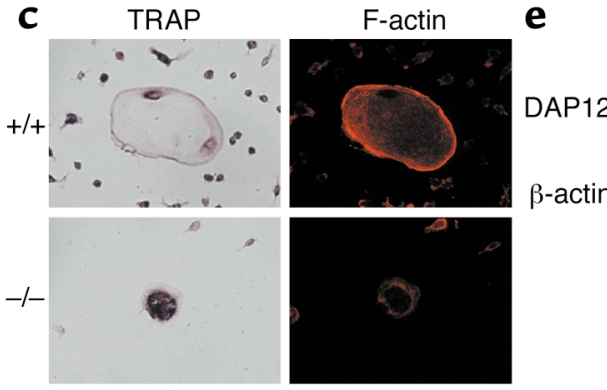



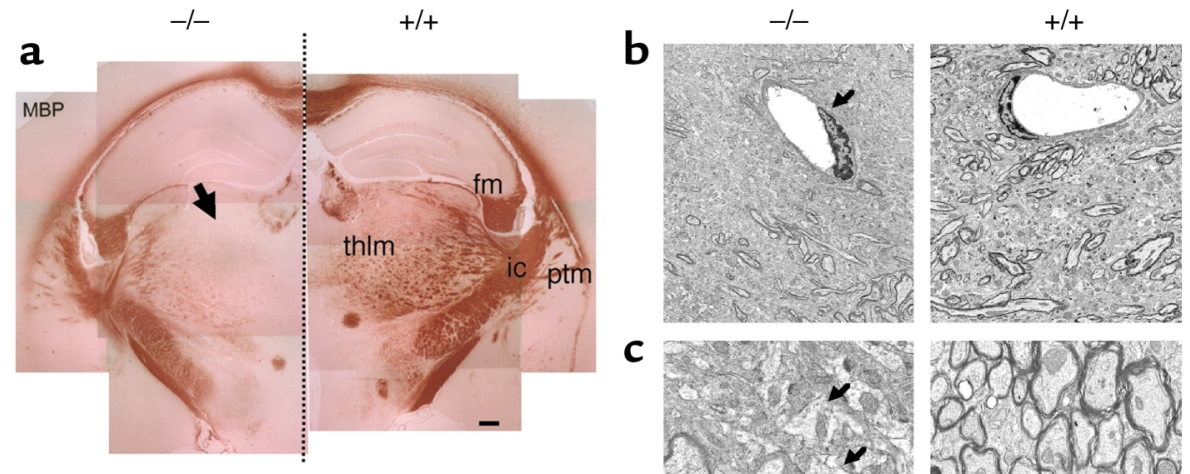

e
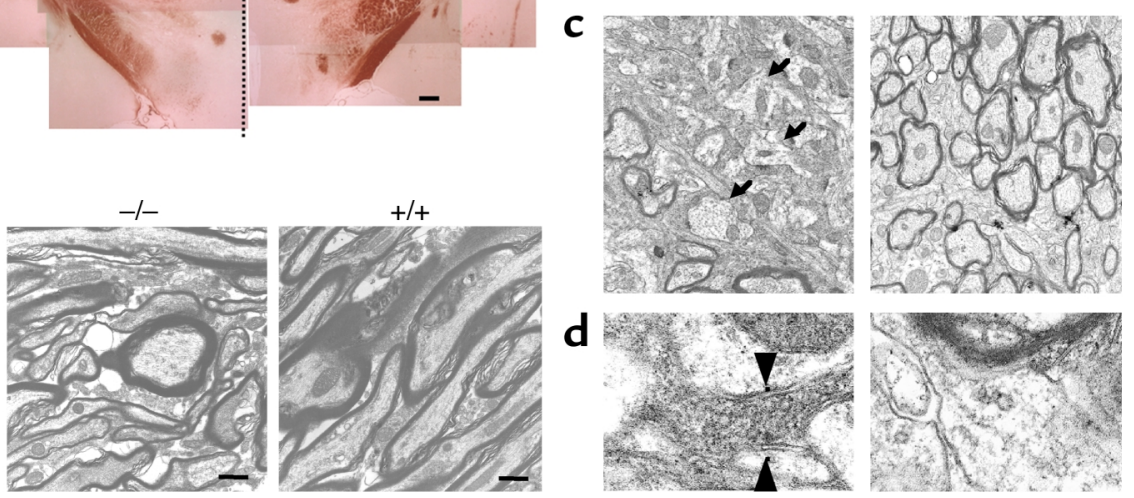

\section{Figure 4}

Thalamus-accentuated hypomyelinosis in DAP12-/- mice. (a) Thalamus-accentuated reduction of MBP staining. Coronal brain sections were stained with anti-MBP antibody. The left side is from DAP12-/- mouse and the right side is from wild-type mouse, both at 3 months of age. Each panel is composed of multiple pictures. Arrow indicates the thalamus-accentuated reduction of MBP staining. fm, fimbria; ic, internal capsule; ptm, putamen; thlm, thalamus. Scale bar, $250 \mu \mathrm{m}$. (b-e) Ultrastructure of medial thalamus (b-d) and putamen (e). (b) Lower-magnification views (original magnification: $\times 2,000$ ) of medial thalamus of 6 -month-old $D A P 12^{-/-}$and wild-type mice. Myelinated axons are reduced significantly in the DAP12-/- mouse, while blood vessels appear to be normal (arrow). (c and d) Higher-magnification views $(\times 10,000$ for $\mathbf{c}, \times 50,000$ for $\mathbf{d}$ ) showing many unmyelinated axons with aberrant morphology (arrows) and electron-dense appearance of the non-axonal area (c), and synaptic degeneration and abnormal accumulation of synaptic vesicles (flanked by arrowheads) in the DAP12-/- mouse (d). (e) Putamen of 3-month-old mice. Hypomyelinosis is not evident in this area, but a somewhat distorted axon shape is evident. Original magnification: $\times 40,000$. Scale bar, $250 \mathrm{~nm}$.

slices from 3- and 12-month-old mice showed no marked differences between wild-type and DAP12-/ mice (data not shown). In addition, there was no significant increase in the number of apoptotic neurons (shown by double staining for Nissl and TUNEL) from a DAP12 $2^{-/}$mouse at 12 months of age (data not shown), eliminating possible impairment in the scavenger effect of microglial cells. Hematoxylin and eosin staining of samples from 12 -month-old $D A P 12^{-/-}$mice showed no evidence of cerebral inflammation (not shown). Instead, we found reduced staining of MBP, a major and specific component of myelin, in the thalamus of DAP12 $2^{-/-}$mice at 1.5 (data not shown) and 3 months of age (Figure 4a), while other areas, such as the putamen, showed no significant difference in MBP staining. In addition, the reduction of MBP staining was more prominent in the frontal thalamus than in the posterior region (data not shown). Myelin enwraps axons and serves as an insulator that is important for the fast conduction of nerve impulses and for axon integrity (29). In the CNS, oligodendrocytes develop myelin. MBP-deficient shi/shi mice show severe hypomyelinosis of CNS axons accompanied by seizure behavior with generalized tremors, culminating in premature death within 3 months (30). It should be noted that many autopsy cases of Nasu-Hakola patients show frontally accentuated loss of myelin together with thalamus degeneration $(2,31)$.

Ultrastructural examination of the medial thalamus of 6-month-old DAP12 $2^{--}$mice revealed a striking hypomyelinosis with some unexpected characteristics (Figure 4, b-d). In lower-magnified views (Figure 4b), the number of myelinated axons was reduced, while endothelial cells and their basement membranes were intact. In the higher-magnified views (Figure 4c), the reduction of myelinated axons was more evident. Many unmyelinated axons were present in the mutant mice, and their cross-sectional shapes were slightly distorted compared with those of myelinated axons in both $D A P 12^{+/+}$and $D A P 12^{-/-}$mice. In addition, many electron-dense regions were observed in the non-axonal area (Figure 4c). The magnified view of the electrondense regions showed a degenerated synapse and accumulated synaptic vesicles (Figure $4 d$ ). On the other hand, the ultrastructure of myelin and axons in the putamen from $D A P 12^{-/-}$mice showed a slightly hypomyelinated appearance with some irregularly shaped axons (Figure 4e). Thus, in the medial thalamus from the adult DAP12-- mouse, we observed severe hypomyelinosis, aberrant cross-sectional morphology of unmyelinated axons, and degenerated synapses containing accumulated synaptic vesicles. 
Oligodendrocytes express DAP12 protein. Although mRNA for DAP12 has been detected in primary cultures of murine microglial cells, astrocytes, and neurons (6), it has not been clarified whether DAP12 protein is expressed in neuronal cells or glial cells, including oligodendrocytes, which are responsible for myelination in the CNS. We prepared primary cultures of these cells in vitro from newborn mice or fetal mouse embryos and subjected them to RT-PCR, Northern blot, and immunoblot analyses. We detected a signal for DAP12 mRNA in $\mathrm{O}^{+}, \mathrm{O}_{4}^{+}$, and $\mathrm{A} 2 \mathrm{~B}^{+}$oligodendrocytes and microglial cells (Figure 5a). In addition, Northern blot analysis revealed a $0.5-\mathrm{kb}$ signal for DAP12 mRNA in oligodendrocytes and microglial cells (Figure $5 \mathrm{~b}$ ). No significant DAP12 mRNA signals were detected in astrocytes or neuronal cells (Figure 5b). Our immunoblot showed a robust signal for DAP12 protein in oligodendrocytes and microglial cells, indicating that these cells express DAP12 protein, although the signal intensities were less significant than those of LAK cells (Figure 5c).

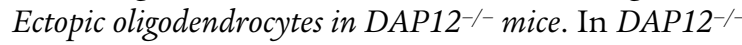
mice 1.5 months old (data not shown) and 3 months old (Figure 5d), many MBP-positive oligodendrocytes were found in the fimbria and internal capsule, sites adjacent to the thalamus. On the other hand, oligodendrocytes in adult brains of wild-type mice could hardly be identified by MBP staining (Figure $5 \mathrm{~d}$ ) due to their development of MBP-positive dendrites. Thus, with DAP12 deficiency, oligodendrocyte development seems to be arrested regionally where myelinogenesis would otherwise have become active. It is possible that DAP12 is required for activation of myelinogenesis in the thalamus and its surrounding area.
Impaired sensorimotor gating in DAP12-/- mice. As noted earlier, $D A P 12^{-/-}$mice did not show any gross behavioral abnormalities even after aging. We did not observe any significant differences between $D A P 12^{-/-}$ and wild-type mice at 3-5 months of age in more detailed analyses of their motor function, nociceptive responses, and learning, including an electric shock test, a hot plate test, and a Morris water maze task (data not shown). However, we found a reduced startle reflex in response to acoustic stimuli in the DAP12- mice (Figure 6a). Moreover, the mutant mice displayed significantly reduced prepulse inhibition (Figure 6b), suggesting an impairment of sensorimotor gating. The acoustic startle reflex is a simple neural circuit that receives input from the thalamus and arises from diverse centers in the CNS. Prepulse inhibition, which is a modulation of the startle response by a weak prepulse, is a measure of sensorimotor gating that refers to the inhibitory process in the CNS by which an animal filters the flow of information via the thalamus and gates its subsequent behavior (Figure 6c). Accordingly, it is possible that $D A P 12^{-/-}$mice have deficits in the proposed neural circuit shown in Figure $6 c$, including an inhibition of thalamus by neurons transmitting GABA (GABAergic neurons, Figure 6c).

We sought to identify any change in electrophysiological properties by recording GABAergic spontaneous mIPSC's in laterodorsal thalamic neurons of DAP12-/- mice. The mIPSC's were reversibly blocked by bath application of bicuculline $(10 \mu \mathrm{M}$, data not shown), suggesting that they were mediated by $\mathrm{GABA}_{\mathrm{A}}$ receptors. The decay time of mIPSC's matched a single

\section{Figure 5}

Expression of DAP12 in oligodendrocytes, and ectopic oligodendrocytes in DAP12-/- mice. (a) RT-PCR analysis for DAP12 mRNA in oligodendrocyte progenitor cells $\left(\mathrm{A}_{2} \mathrm{~B} 5^{+}, \mathrm{O}_{4}^{+}\right.$, or $\mathrm{O} 1^{+}$cells $)$and microglial cells prepared from wild-type mice. LAK cells from wild-type mice presented as a positive control. Oligodendrocyte precursor cells are positive for mRNA of DAP12 as well as proteolipid protein (PLP) and its splice variant, DM20, marker proteins for oligodendrocytes. (b) Northern blot analysis of DAP12 mRNA in neuron, astrocyte, oligodendrocyte, and microglia. Thirty micrograms (neuron, astrocyte, and oligodendrocyte) or $5 \mu \mathrm{g}$ (microglia) of total RNA was subjected to electrophoresis on a $1.2 \%$ agarose gel containing formaldehyde, transferred to nylon membrane, and probed with $[\alpha-32 P] d C T P-l a b e l e d$, full-length coding region of DAP12 cDNA. Oligodendrocytes and microglia expressed a significant amount of DAP12 mRNA. (c) Immunoblot analysis of DAP12. Oligodendrocytes and microglia express DAP12 protein, although the expression levels were less significant than those in LAK cells. (d) Staining for MBP of the internal capsule (ic) and thalamus (thlm) from 3-month-old DAP12-/- (upper left and lower) and wild-type (upper right) mice. Ectopic, MBP-positive oligodendrocyte clusters were observed in DAP12-/- mice. Scale bars, $100 \mu \mathrm{m}$. a
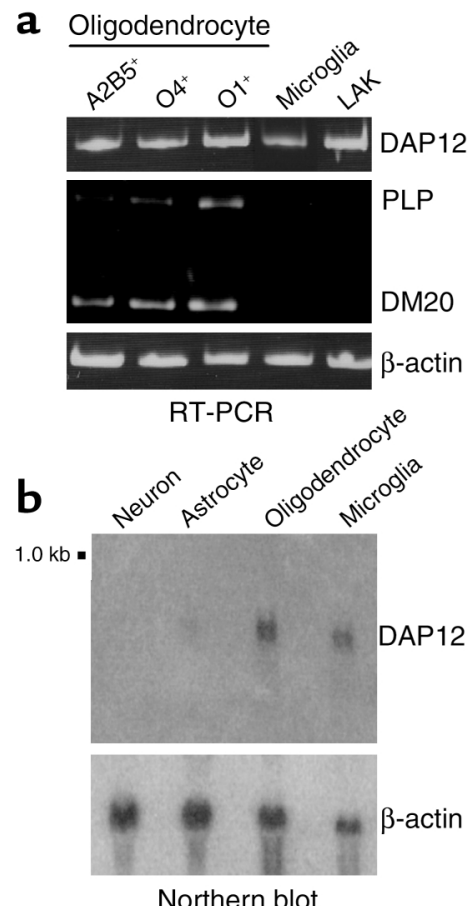

Northern blot
C
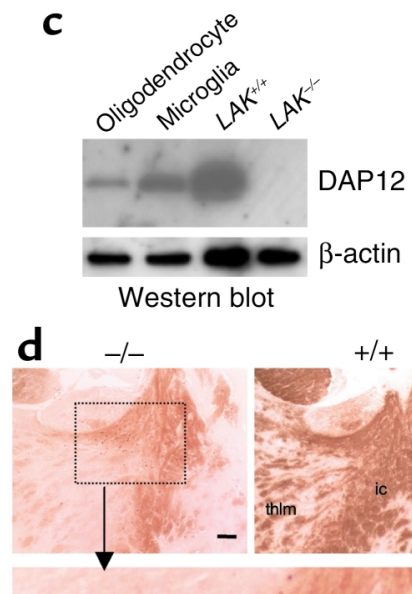

$-1-$

$+1+$

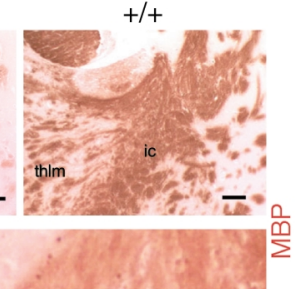


a

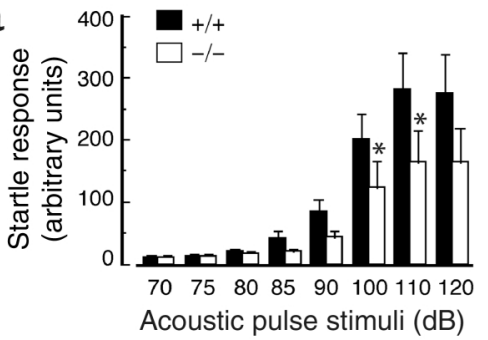

c

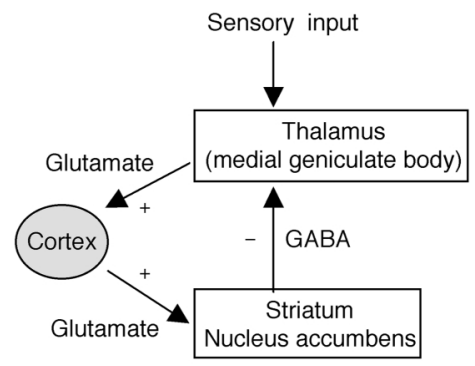

b

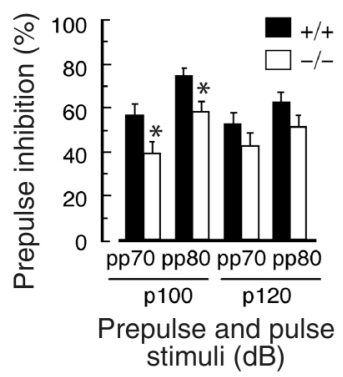

d

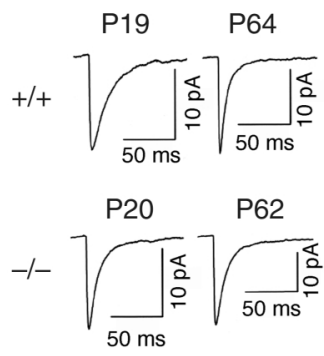

\section{Figure 6}

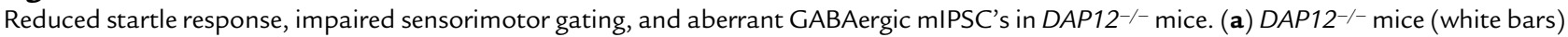
displayed significantly lower startle responses than did wild-type mice (black bars). $\mathrm{F}(1,18)=5.790, P<0.05$. The average startle responses at 100 and $110 \mathrm{~dB}$ were significantly lower in knockout mice. (b) $D A P 12^{-/-}$mice (white bars) displayed significantly lower prepulse (pp) inhibition than did wild-type mice (black bars). $F(1,18)=5.061, P<0.05$. This effect was attributable to the acoustic startle pulse ( $p$ ) stimulus of $100 \mathrm{~dB}$. In $\mathbf{a}$ and $\mathbf{b}$, the data are presented as mean $\pm \operatorname{SEM}\left(n=10,{ }^{*} P<0.05\right)$. (c) Schematic illustration of a neural circuit for negative feedback giving rise to prepulse inhibition. The thalamus receives inhibition by GABAergic neurons. (d) Developmental change in the decay time constant of spontaneous mIPSC's recorded from laterodorsal thalamic neurons in the presence of tetrodotoxin; 6-cyano-7-nitroquinoxaline2,3-dione; $\mathrm{D}(-)-2$-amino-5-phosphonopentanoic acid; and strychnine. The mean decay time constants of mIPSC's recorded from DAP12 ${ }^{-/-}$ mice (open circles) were $12.2 \pm 0.7 \mathrm{~ms}(n=5)$ and $10.9 \pm 0.2 \mathrm{~ms}(n=4)$ at P20 and P62, respectively, whereas those from wild-type mice (filled circles) were $17.3 \pm 1.1 \mathrm{~ms}(n=4)$ and $8.1 \pm 1.0 \mathrm{~ms}(n=4)$ at $\mathrm{P} 19$ and $\mathrm{P} 64$, respectively. The numbers of neurons tested are given in parentheses. The decay time matched a monoexponential time course. The current traces on the left are the averaged mIPSC's (from 491-999 events) at each age of wild-type or DAP12-/- mice.

exponential time course as previously reported (32). In wild-type mice, the decay time of mIPSC's became faster as the animal matured, in agreement with the previous report on rats (32). The mean decay time constant of mIPSC's was $17.3 \pm 1.2 \mathrm{~ms}$ (mean \pm SEM, $n=4$ cells) at postnatal day 19 (P19), whereas it was $8.1 \pm 1.0$ ms $(n=4)$ at $\mathrm{P} 64$, significantly different $(P<0.05)$ from at P19 in wild-type mice (Figure $6 \mathrm{~d}$ ). On the other hand, in DAP12-/- mice, the developmental shortening of decay time was not significant $(P>0.05)$. The mean decay time constant of mIPSC's in DAP12 ${ }^{-/-}$mice was $12.2 \pm 0.7 \mathrm{~ms}(n=5)$ and $10.9 \pm 0.2 \mathrm{~ms}(n=4)$ at P20 and P62, respectively (Figure 6d). Thus, impairment in developmental changes of the decay time constant in GABAergic mIPSC's of DAP12-/- mice was observed. It is possible that the diminished shortening of the decay time constant in $\mathrm{DAP} 12^{-/-}$mice relates to the reduced startle response and impaired prepulse inhibition.

\section{Discussion}

Osteopetrosis results in excessively dense bones, and the bones become hard, but are brittle and more often fractured than normal bones (25). This notion may link, at least in part, Nasu-Hakola disease to osteopetrosis.
Nasu-Hakola patients usually exhibit pain and swelling of the wrist and ankle at age 20-30, and fractures sometimes occur after minor accidents, although these skeletal symptoms are often mild and in most cases the diagnosis is made after the onset of neuropsychiatric symptoms (5). Osteopetrosis in DAP12 $2^{-/-}$mice was relatively mild compared with that observed in Csf 1 op/op and other osteopetrotic mutant mice $(24,25)$. Although the ultrastructure of osteoclasts in DAP12/- mice in vivo was not grossly altered, they had reduced bone remodeling activity, as demonstrated in in vitro studies. We do not know the reason why the DAP12 $2^{-/-}$osteoclasts found in vivo and in vitro were different, particularly in morphology. One plausible explanation is that in vivo, the DAP12 $2^{-/}$osteoclasts may be able to differentiate mainly via other signaling adapters harboring ITAM, such as the Fc receptor $\gamma$ subunit. The ITAM-mediated activation signaling initiated by unidentified receptors and their ligands may have a redundant role for osteoclast differentiation and maturation in vivo. Detailed comparison of the roles of DAP12 and Fc receptor $\gamma$ chain in osteoclast signaling will clarify this point.

The mechanism by which thalamic hypomyelinosis occurs in DAP12 $2^{-/-}$mice remains to be elucidated. It is 
most plausible that DAP12-/- oligodendrocytes do not develop properly in the thalamus and cannot maturate in terms of building myelin structures; this notion is supported by the fact that DAP12-/- oligodendrocytes accumulated in the vicinity of the thalamus. If this is the case, the aberrant axonal morphology and synaptic degeneration may be a secondary effect after the primary hypomyelination of axons. Alternatively, considering the DAP12 expression in microglia and oligodendrocytes, the hypomyelinosis and synaptic degeneration could be the result of a combined defect of these cell types. In either case, we also do not know the reason why the hypomyelinosis occurs in a thalamus-accentuated fashion. It is probable that the ligand for TREM2 or other unknown receptors associating with DAP12 may locate in the thalamus or its vicinity.

The present electrophysiological results demonstrate that the shortening of mIPSC decay time observed in $D A P 12^{+/+}$mice or rats (32) is absent in DAP12 $2^{-/-}$mice. It has been suggested that the prolongation of decay time can cause sedation $(33,34)$, which might lead to the reduced startle response or impaired sensorimotor gating observed in the present behavioral studies. It has also been suggested that the duration of GABAergic IPSC's is an important factor regulating the thalamocortical activity underlying the waking, slow-wave sleep and generation of seizures (35), and that developmental shortening of the IPSC decay time contributes to maintaining a high level of consciousness in mature animals (32). Therefore, DAP12 could be one of the key molecules required for maintaining such higher CNS functions.

Impairment in sensorimotor gating results in sensory inundation, cognitive fragmentation, and attention deficits, as seen in several human neuropsychiatric disorders including schizophrenia, schizotypal personality disorders, and Tourette syndrome (36-39). The molecular mechanisms underlying the defects of schizophrenia and the related disorders remain unclear. Thalamic abnormalities in schizophrenia have been observed in a study using magnetic resonance image (MRI) averaging (40). Of note, DNA microarray analysis very recently suggested a disruption in oligodendrocyte function in schizophrenia patients (41). In Nasu-Hakola patients, schizophrenic symptoms such as personality change and loss of social inhibitions have been observed before the development of presenile dementia. In fact, an MRI of one Nasu-Hakola patient found reduced signal intensity in the thalamus and putamen (3). It is possible that some schizophrenia patients may have defects in myelinogenesis in their thalamus due to impaired oligodendrocyte development.

How do Nasu-Hakola patients develop early-onset dementia? It is possible that the damaged neuronal cells in the patient's thalamus gradually die by apoptosis or by immunological attack. In adult $D A P 12^{-/-}$mice, however, we detected neither apoptotic neuronal cells nor any evidence of inflammation. Therefore, neuronal cells in mice may be more resistant to the hypomyelinosis than those in humans. Identification of the full lineup of the common adapter molecules of the immune system and the CNS, along with their associated receptors and their physiological ligands, and the precise mapping of each in the CNS, will provide us with the keys to identify the onset mechanisms of schizophrenia and presenile dementia in humans.

\section{Acknowledgments}

We thank M. Obinata for critical reading of the manuscript; M. Ogawa, N. Aoki, N. Toyama-Sorimachi, and H. Karasuyama for reagents; R. Hammer and Y. Koyanagi for helpful advice; and Y. Ishikawa, K. Maya, and Y. Ito for technical support. This work is supported by the CREST program of Japan Science and Technology Corp.; a Grant-in-Aid from the Ministry of Education, Culture, Sports, Science and Technology of Japan; Ono Medical Research Foundation; and by the Virtual Research Institute of Aging funded by Nippon Boehringer Ingelheim Co. (to T. Takai).

1. Nasu, T., Tsukahara, Y., and Terayama, K. 1973. A lipid metabolic disease-"membranous lipodystrophy"-an autopsy case demonstrating numerous peculiar membrane-structures composed of compound lipid in bone and bone marrow and various adipose tissues. Acta Pathol. Jpn. 23:539-558.

2. Hakola, H.P.A. 1972. Neuropsychiatric and genetic aspects of a new hereditary disease characterized by progressive dementia and lipomembranous polycystic osteodysplasia. Acta Psychiatr. Scand. 232(Suppl.):1-173.

3. Verloes, A., et al. 1997. Nasu-Hakola syndrome: polycystic lipomembranous osteodysplasia with sclerosing leukoencephalopathy and presenile dementia. J. Med. Genet. 34:753-757.

4. Tanaka, J. 2000. Nasu-Hakola disease: a review of its leukoencephalopathic and membranolipodystrophic features. Neuropathology. 20:S25-S29.

5. Paloneva, J., et al. 2001. CNS manifestations of Nasu-Hakola disease: a frontal dementia with bone cysts. Neurology. 56:1552-1558.

6. Paloneva, J., et al. 2000. Loss-of-function mutations in TYROBP (DAP12) result in a presenile dementia with bone cysts. Nat. Genet. 25:357-361.

7. Olcese, L., et al. 1997. Killer-cell activatory receptors for MHC class I molecules are included in a multimeric complex expressed by human killer cells. J. Immunol. 158:5083-5086.

8. Lanier, L.L., Corliss, B.C., Wu, J., Leong, C., and Phillips, J.H. 1998. Immunoreceptor DAP12 bearing a tyrosine-based activation motif is involved in activating NK cells. Nature. 391:703-707.

9. Daws, M.R., Lanier, L.L., Seaman, W.E., and Ryan, J.C. 2001. Cloning and characterization of a novel mouse myeloid DAP12-associated receptor family. Eur. J. Immunol. 31:783-791.

10. Bouchon, A., Hernández-Munain, C., Cella, M., and Colonna, M. 2001. A DAP12-mediated pathway regulates expression of CC chemokine receptor 7 and maturation of human dendritic cells. J. Exp. Med. 194:1111-1122.

11. Bakker, A.B.H., et al. 2000. DAP12-deficient mice fail to develop autoimmunity due to impaired antigen priming. Immunity. 13:345-353.

12. Tomasello, E., et al. 2000. Combined natural killer cell and dendritic cell functional deficiency in KARAP/DAP12 loss-of-function mutant mice. Immunity. 13:355-364.

13. Aoki, N., et al. 2000. The role of the DAP12 signal in mouse myeloid differentiation. J. Immunol. 165:3790-3796.

14. Paloneva, J., et al. 2002. Mutations in two genes encoding different subunits of a receptor signaling complex result in an identical disease phenotype. Am. J. Hum. Genet. 71:656-662.

15. Takai, T., Li, M., Sylvestre, D., Clynes, R., and Ravetch, J.V. 1994. FcR $\gamma$ chain deletion results in pleiotrophic effector cell defects. Cell. 76:519-529.

16. Seiwa, C., Sugiyama, I., Yagi, T., Iguchi, T., and Asou, H. 2000. Fyn tyrosine kinase participates in the compact myelin sheath formation in the central nervous system. Neurosci. Res. 37:21-31.

17. Knapp, P.E., Skoff, R.P., and Sprinkle, T.J. 1988. Differential expression of galactocerebroside, myelin basic protein, and $2^{\prime}, 3^{\prime}$-cyclic nucleotide $3^{\prime}$-phosphohydrolase during development of oligodendrocytes in vitro. J. Neurosci. Res. 21:249-259. 
18. Bottenstein, J.E. 1986. Growth requirement in vitro of oligodendrocyte cell lines and neonatal rat brain oligodendrocytes. Proc. Natl. Acad. Sci. USA. 83:1955-1959.

19. Raizada, M.K., Yang, J.W., and Follows, R.E. 1980. Binding of $\left[{ }^{125} \mathrm{I}\right]$ insulin to specific receptors and stimulation of nucleotide incorporation in cells cultured from rat brain. Brain Res. 200:389-400.

20. Matsuda, Y., Koito, H., and Yamamoto, H. 1997. Indication of myelinassociated glycoprotein expression through neuron-oligodendrocyte contact. Dev. Brain Res. 100:110-116.

21. Momiyama, T., and Koga, E. 2001. Dopamine $\mathrm{D}_{2}$-like receptors selectively block $\mathrm{N}$-type $\mathrm{Ca}^{2+}$ channels to reduce GABA release onto rat striatal cholinergic interneurones. J. Physiol. (Lond.) 533:479-492.

22. Chang, C., et al. 1999. KAP10, a novel transmembrane adapter protein genetically linked to DAP12 but with unique signaling properties. J. Immunol. 163:4651-4654.

23. Wu, J., et al. 1999. An activating immunoreceptor complex formed by NKG2D and DAP10. Science. 285:730-732.

24. Yoshida, H., et al. 1990.The murine mutation osteopetrosis is in the coding region of the macrophage colony stimulating factor gene. Nature. 345:442-444.

25. McLean, W., and Olsen, B.R. 2001. Mouse models of abnormal skeletal development and homeostasis. Trends Genet. 17:S38-S43.

26. Kodama, H., Nose, M., Niida, S., and Yamasaki, A. 1991. Essential role of macrophage colony-stimulating factor in the osteoclast differentiation supported by stromal cells. J. Exp. Med. 173:1291-1294.

27. Kong, Y.Y., et al. 1999. OPGL is a key regulator of osteoclastogenesis, lymphocyte development and lymph-node organogenesis. Nature. 397:315-323.

28. Azuma, Y., Kaji, K., Katogi, R., Takeshita, S., and Kudo, A. 2000. Tumor necrosis factor- $\alpha$ induces differentiation of and bone resorption by osteoclasts. J. Biol. Chem. 275:4858-4864.

29. Griffiths, I., et al. 1998. Axonal swelling and degeneration in mice lacking the major proteolipid of myelin. Science. 280:1610-1613.

30. Duponey, P., et al. 1979. Immunochemical studies of myelin basic pro- tein in shiverer mouse devoid of major dense line of myelin. Neurosci. Lett. 12:113-118.

31. Kobayashi, K., et al. 2000. Hypothalamic haemorrhage and thalamus degeneration in a case of Nasu-Hakola disease with hallucinatory symptoms and central hypothermia. Neuropathol. Appl. Neurobiol. 26:98-101.

32. Okada, M., Onodera, K., van Renterghem, C., Sieghart, W., and Takahashi, T. 2000. Functional correlation of $\mathrm{GABA}_{\mathrm{A}}$ receptor $\alpha$ subunits expression with the properties of IPSCs in the developing thalamus. J. Neurosci. 20:2202-2208.

33. Tanelian, D.L., Kosek, P., Mody, I., and MacIver, M.B. 1993. The role of the $\mathrm{GABA}_{\mathrm{A}}$ receptor/chloride channel complex in anesthesia. Anesthesiology. 78:757-776

34. Franks, N.P., and Lieb, W.R. 1994. Molecular and cellular mechanisms of general anesthesia. Nature. 367:607-614.

35. Kim, U., Sanchez-Vives, M.V., and McCormick, D.A. 1997. Functional dynamics of GABAergic inhibition in the thalamus. Science. 278:130-134.

36. Braff, D.L., et al. 2001. Impact of prepulse characteristics on the detection of sensorimotor gating deficits in schizophrenia. Schizophr. Res. 49:171-178.

37. Romanski, L.M., and LeDoux, J.E. 1992. Equipotentiality of thalamoamygdala and thalamo-cortico-amygdala circuits in auditory fear conditioning. J. Neurosci. 12:4501-4509.

38. Wu, M.F., Mallick, B.N., and Siegel, J.M. 1989. Lateral geniculate spikes, muscle atonia and startle response elicited by auditory stimuli as a function of stimulus parameters and arousal state. Brain Res. 499:7-17.

39. Kodsl, M.H., and Swerdlow, N.R. 1997. Regulation of prepulse inhibition by ventral pallidal projections. Brain Res. Bull. 43:219-228.

40. Andreasen, N.C., et al. 1994. Thalamic abnormalities in schizophrenia visualized thorough magnetic resonance image averaging. Science. 266:294-298.

41. Hakak, Y., et al. 2001. Genome-wide expression analysis reveals dysregulation of myelination-related genes in chronic schizophrenia. Proc. Natl. Acad. Sci. USA. 98:4746-4751. 